\title{
ASPECTOS ONTOLÓGICOS Y EPISTEMOLÓGICOS DE LAS VISIONES DE ENFERMERÍA INMERSAS EN EL QUEHACER PROFESIONAL
}

\section{ONTOLOGICAL AND EPISTEMOLOGICAL ASPECTS OF THE NURSING VIEWS IMMERSED IN THE PROFESSIONAL WORK}

\author{
Luz Stella Bueno Robles*
}

\begin{abstract}
RESUMEN
La enfermería ha avanzado no sólo por la indagación científica, sino por los aportes de la filosofía en los aspectos ontológicos y epistemológicos presentes en las visiones de enfermería como: la reactiva o determinística, de reciprocidad o integrativa y la simultánea o unitaria transformativa, que han impregnado el quehacer profesional generando cambios trascendentales en conceptos como el ser humano o persona, ambiente y relaciones, el método de investigación, el cuidado y la valoración del paciente. El objetivo del presente artículo es describir, desde la postura presentada por las tres visiones, los aspectos de origen de la visión, la concepción de ser humano o persona, ambiente y relaciones, el método de investigación, el cuidado y la valoración del paciente acorde a su orientación filosófica, con el fin de mostrar un acercamiento entre el pensamiento abstracto y la realidad práctica de enfermería.
\end{abstract}

Palabras clave: Enfermería, conocimiento, epistemología, práctica profesional, filosofía.

\begin{abstract}
The nursing evolution has advanced not only for the scientific research but for the contributions of the philosophy in the ontological and epistemological aspects arising in the nursing view like the reaction or deterministic, reciprocity or integrative and simultaneous or transformative unitary, which have impregnated the professional task generating decisive changes in concepts like the human being or person, environment and relationships, the research method, the care and the assessment of the patient. The aim of the present article is to describe the position presented by three visions: the aspects of origin of the vision, the conception of human being, environment and relations, the method of research, the care and the evaluation's patient related with philosophical orientation, to show a bridge between abstract thought and reality of nursing practice.
\end{abstract}

Key words: Nursing, knowledge, epistemology, professional practice, philosophy.

Fecha recepción: 31/03/2010 Fecha aceptación: 13/04/2011

\section{INTRODUCCIÓN}

Las visiones de mundo son formas abstractas de ver la realidad y reflejan las pretensiones que desde el punto de vista ontológico (ser) y epistémico (conocer) tienen las diferentes disciplinas, en este caso en parti- cular, la enfermería a través de los años ha estado permeada por diferentes momentos histórico-culturales y corrientes filosóficas que han contribuido al desarrollo del conocimiento, la práctica y la investigación. Butts y Rich plantean que la revisión previa de las novedades y tendencias en la evolución de enfermería ofrece un vistazo a los progresos

\footnotetext{
* Doctora@ en Enfermería Universidad Nacional de Colombia. Becaria del Instituto Colombiano para el Desarrollo de la Ciencia y la Tecnología, COLCIENCIAS. Email: stellita2008@hotmail.com.
} 
realizados en la disciplina que se han enfocado a: 1) la generación de una base sólida de conocimientos y 2) al fortalecimiento de la identidad. También, destacan que "numerosos conocimientos sean recreados dentro de la disciplina, pero algunos fueron tomados como recetas para el pensamiento de enfermería; mientras que la variedad de enfoques filosóficos y metodológicos demandan de la enfermera un avance en el rol de la práctica" $(1,2)$. Se requiere entender que la evolución de enfermería ha avanzado no sólo por la indagación científica sino por los aportes de la filosofía en los aspectos ontológicos y epistemológicos emergentes en las visiones de enfermería. Entonces, surge la necesidad de centrarnos en la importancia de la influencia filosófica en el quehacer del profesional, porque a través de esta área del conocimiento podemos encontrar múltiples formas para acercarnos de manera diversa al "otro" y comprender así los posibles significados que las personas dan a las experiencias y realidades (3). Monti (4) señala que la enfermería como disciplina tiene dos visiones: la empirista y la interpretativa, en tanto que Fawcett (5) y Newman (6) consideran que son tres: de reacción o particular determinística, de reciprocidad o interactiva integrativa y simultánea o unitaria transformativa. El objetivo del presente artículo es describir desde la postura presentada por las tres visiones los aspectos de origen de la visión, la concepción de ser humano o persona, ambiente y relaciones, el método de investigación, el cuidado y la valoración del paciente acorde a su orientación filosófica.

\section{Visión reactiva o particular determinís-} tica. La visión reactiva o particular determinística surge del positivismo, que es una corriente filosófica que concibe la ciencia como la descripción pura de los hechos y se identifica con la verdad demostrada a través de hechos empíricamente verificables (7), en este caso, el mundo y la realidad son medibles y cuantificables. Está visión también se origina del mecanicismo, que interpreta la realidad como una máquina o modelo mecánico regido por leyes que regulan los fenómenos, facilitan la exploración de causas-efectos y por ende predicen resultados $(8,9)$. El positivismo lógico aseguró que la única realidad era la que podía ser observada (empirismo) y razonada (racionalismo) $(10,11)$, como lo refieren Jacox y Webster, si se usan los lentes de la visión reactiva "el universo es visto como una gran máquina operada por leyes que sólo son entendidas por Dios" (12). Las personas desde esta perspectiva son seres pasivos y fraccionados (8) que no interactúan con el medio externo, se mantienen estables, sólo responden a estímulos y cambian sólo cuando reaccionan para sobrevivir. Desde esta mirada, los seres humanos son la suma de partes discretas, lo que conlleva a que enfermería desarrolle la práctica, de forma aislada, dividiendo sus intervenciones y valoraciones para suplir las necesidades o requerimientos en las áreas en las cuales se cree está divido el ser: biológica, social, emocional y espiritual. No es posible desde el determinismo ir más allá de lo que se evidencia, dado que la enfermedad o deficiencia establece la actuación y el resultado a esperar, por lo que el propósito será corregir las causas o etiologías de los cambios que se están desarrollando en las diferentes partes que constituyen al ser humano. En la práctica esto se relaciona con intervenciones puntuales, medibles que se orientan a la recuperación posiblemente de la función de órganos o sistemas. El ambiente y las relaciones con el medio externo producen estímulos que generan reacciones en el ser humano (8), desde esta orientación paradigmática, el profesional de enfermería es el agente encargado de realizar todas las acciones de cuidado para generar cambios; se ve a las personas como seres pasivos, incapaces de realizar por sí mismos acciones que le permitan lograr la salud o bienestar. Florence Nightingale (13) coincide con las lineamientos de esta visión y define a la en- 
fermera como la persona con conocimientos teóricos y habilidades prácticas para observar inteligentemente a los pacientes y el entorno, mientras que el paciente es visto como un ser pasivo. Por otra parte, el conocimiento es desarrollado por el método científico con un abordaje cuantitativo caracterizado por la medición, objetividad y control (8) a través de diseños experimentales que buscan identificar las causas y efectos del cuidado de enfermería. Con respecto, al conocimiento científico desarrollado dentro de la visión reactiva es limitado porque se centra en objetividad y observación (8) de los seres humanos, olvidando la interpretación de la subjetividad que permite entender y contextualizar el cuidado. Se utiliza el proceso de enfermería (14): valoración (por patrones, sistemas, dominios), diagnóstico, planeación, ejecución de las intervenciones y evaluación (15), para brindar atención a todas los personas. Es importante, resaltar que el proceso de enfermería es una herramienta fundamental para el desarrollo del cuidado porque provee las pautas a seguir y organiza el cuidado que se brinda a las personas, sin embargo, la debilidad está en convertirlo en una actividad mecánica, colectiva y descontextualizada al momento de implementarlo.

\section{Visión interacción recíproca o interactiva} integrativa. Se origina del postpositivismo que da relevancia al ser humano, al contexto e historicidad e incluye los valores, las creencias, intereses y experiencias (8). La visión interacción recíproca está representada por el organicismo como forma de interpretar el mundo y los seres caracterizados por el cambio, la persistencia y la interacción. El ser humano es holístico, integrado, organizado y no reducible (8) a sus partes, conllevando a que enfermería brinde atención a las personas de una manera integral porque no sólo cuidan un cuerpo enfermo físicamente sino que reconocen la importancia e identifican los demás aspectos que pueden estar afecta- dos como el psicológico, social y espiritual. Callista Roy (16) define a las personas como sistemas holísticos que constituyen el foco de atención de enfermería y son los receptores de la asistencia. Los cambios enfrentados por las personas pueden ocurrir simultáneamente, es decir, en cualquier momento de la vida, a veces son continuos, persistentes, se pueden estimar, pero no predecir. Esta visión considera que las personas son seres cambiantes que enfrentan diariamente situaciones que afectan o mejoran los procesos de salud. Cuidar en enfermería implica un conocimiento propio de cada enfermo, un darse cuenta de sus actitudes, aptitudes y motivaciones y además, de sus conocimientos requiere su manifestación como persona única, auténtica, capaz de generar confianza, serenidad, seguridad y apoyo efectivo (17). El conocimiento disciplinar en esta visión es desarrollado a través de abordajes cualitativos y cuantitativos que se complementan y aportan resultados científicos para fundamentar el cuidado. Por su parte, la investigación cualitativa usa métodos fenomenológicos para descubrir, explicar y comprender situaciones específicas de la vida, mientras que los estudios cuantitativos desarrollan generalmente estudios correlacionales para explicar y predecir los fenómenos de interés (18). Los profesionales que se orientan por la visión recíproca, manejan estos dos tipos de abordajes y los implementan de acuerdo a la temática y vacíos del conocimiento que se quiere explorar; se determina si se realiza investigación cualitativa o cuantitativa, en este caso "se reconoce la pluralidad como único camino de construcción de un conocimiento en salud y en enfermería" (19). Los resultados de los estudios deben aportar al cuidado de enfermería que es considerado como una ciencia y arte que exige conocimiento y actitudes especiales fundamentadas (20). Lo anterior destaca la importancia de los aportes de la investigación en enfermería, dado que permite generar y probar teorías relacionadas con la salud de los seres humanos dentro 
de sus entornos y demostrar las acciones y procesos que enfermería requiere aplicar en la práctica $(21,22)$.

\section{Visión simultánea o unitaria transforma-} tiva. La visión simultánea surge a partir de la combinación de elementos del organicismo, simultaneidad, cambio y unitario transformativo (8) que constituyen una forma característica de interpretar el mundo y los seres como una organización que evoluciona a través de la historia. La filosofía del pensamiento se fundamenta en corrientes postpositivistas como: la fenomenología, hermenéutica, humanismo, idealismo y existencialismo (23). Las personas son seres unitarios, campos holísticos, autoorganizados e irreducibles que responden simultáneamente a estímulos; esta misma visión es asumida por Martha Rogers (16) en su teoría "seres humanos unitarios” e identifica a la persona como un todo unido que posee integridad y características evidentes, que lo convierten en algo más que la suma de las partes que lo componen. Esta connotación permite ver al ser humano como único, diferente y organizado por patrones; por ejemplo, si se tienen varios pacientes postquirúrgicos, cada uno va a presentar diferentes niveles de dolor; en esta situación, la enfermera entiende los diferentes patrones de comportamiento $y$ reconoce, por ejemplo, que el dolor no sólo afecta una parte del cuerpo sino que afecta la organización total de la persona. La visión simultánea considera el intercambio personal y ambiente como un proceso mutuo y rítmico en donde se generan transacciones que permiten intercambiar continuamente con el medio externo (8). Rogers plantea que las personas son sistemas abiertos en proceso continuo con el sistema abierto que es el entorno, a sí mismo define el patrón como algo abstracto que se revela por medio de comportamientos, cualidades y características del campo $(5,16)$. El método utilizado para desarrollar el conocimiento es el cualitativo que estudia los fenómenos humanos en ambientes naturales a partir del punto de vista holístico (24) buscando explorar la realidad interna. "La enfermería se ha apropiado de esa aproximación para entender la historia de las personas, sus condiciones de vida y salud, sentimientos, creencias y experiencias para interpretar el contexto investigado" (25). Para identificar los patrones de comportamiento y la subjetividad del ser humano implementa dos metodologías como: 1. Fenomenología que busca describir las experiencias, tal y como son vividas, experimentadas por el sujeto, en términos de un fenómeno particular. La fenomenología es a la vez una filosofía y un método y en ambos casos puede orientar la investigación de enfermería para contestar preguntas relacionadas con los fenómenos que son experimentados como parte del cuidado de la experiencia de la salud humana (26); 2. Hermenéutica, orienta la comprensión e interpretación de una teoría o una narración (27, 9). Los profesionales de la visión simultánea desarrollan investigación cualitativa que permite explorar la subjetividad de las personas, permitiendo contextualizar y entender los significados de los diversos procesos y situaciones de la vida. El paradigma cualitativo refleja la necesidad de buscar la totalidad del fenómeno, aumentando las relaciones entre las ciencias de la salud - las ciencias humanas y la perspectiva del cuidado de la vida y no sólo de la persona $(25,28)$. Sin embargo, el utilizar sólo este método dificulta el desarrollo de la disciplina porque la investigación se orienta sólo en aspectos cualitativos y deja a un lado otros elementos que deben ser medidos a través del abordaje cuantitativo; éste último es necesario para mostrar el impacto del cuidado de enfermería en estos momentos álgidos en que la sociedad exige resultados y competitividad profesional. Por otra parte, el cuidado de enfermería en esta visión es conceptualizado como un rasgo humano que motiva a acciones integradas en un todo, en donde el profesional de enfermería desarrolla empatía con la persona que cuida, se 
genera retroalimentación durante el cuidado dando como resultado un cuidado individualizado y permitiendo la participación activa y continua del paciente, como lo resaltan Daza, Torres y Prieto (29), "la participación del paciente en el cuidado hace mucho más humana la práctica de enfermería debido a que permite reconocer al otro como persona, con el derecho y la obligación de ser actor de su salud y no simplemente el receptor de actividades planeadas por el profesional que lo atiende". Otra concepción importante del cuidado es que retoma el término "cuidado congruente" porque considera que las acciones y actividades deben estar dirigidas a asistir, apoyar y facilitar a un individuo o grupo con necesidades evidentes o anticipadas para mejorar su condición humana o estilo de vida (30). La práctica guiada desde la simultaneidad valora fundamentalmente las narrativas, las vivencias y experiencias de lo que representa para la persona la situación. El profesional que tenga esta orientación en su quehacer reflejará afecto, interés genuino por lo que ocurre con el otro, las acciones de cuidado serán de tipo sanador. Por último, la valoración de enfermería no utiliza instrumentos, sino que permite al paciente compartir sus experiencias y sentimientos con el fin de comprender y ofrecer un cuidado más apropiado a las necesidades, por lo que se requiere de experticia y compromiso para identificar las necesidades de las personas.

\section{CONSIDERACIONES FINALES}

Las diferencias de las tres visiones son sustantivas, desde el punto de vista ontológico la concepción del ser humano no es la misma, ni cercana una de otra, lo que señala diferencia en las formas de interacción que se derivan, las intervenciones y los cuidados que enfermería ofrece. Desde el punto de vista epistemológico lo que se desea conocer es diferente y las orientaciones teóricas y me- todológicas utilizadas en la indagación también son distintas. La objetividad con que se aborda la investigación en el tipo particular determinística, señala la separación del objeto estudiado y el investigador, en tanto que la visión unitaria transformativa fundamentada en la interrelación entre el investigador y los investigados, da lugar a la intersubjetividad, que es la que permite el desarrollo de relaciones de cuidado verdaderas, significativas y con contenido. El quehacer de los profesionales se enmarca en una visión filosófica que corresponde de alguna manera a las tres visiones que han sido identificadas dentro de la disciplina; se resalta que la mayoría de las acciones pueden estar inmersas dentro de una visión, pero esto no limita el movimiento dentro de las visiones según los requerimientos del cuidado que se necesita. Es necesario que enfermería promueva el reconocimiento de los aportes ontológicos y epistemológicos de las visiones como formas de observar la realidad y fundamentar la disciplina de enfermería, de tal forma que se genere un mayor acercamiento entre el pensamiento abstracto y la realidad práctica del profesional.

\section{REFERENCIAS}

1. Butts JB, Rich KL. Philosophies and theories for advanced nursing practice. Estados Unidos: Jones \& Bartlett Learning; 2011.

2. Parker M. Nursing theories and nursing practice. Philadelphia: F.A Davis Company; 2001.

3. Valverde C. Enfermería centrada en los significados del paciente: La importancia de la filosofía para enfermería. Norte de Salud Mental [Revista en Internet] 2007. Acceso el 12 de abril 2011; 7(28). Hallado en: http://www.ome-aen.org/ NORTE/28/NORTE_28_030_8-15.pdf.

4. Monti EJ, Tingen MS. Multiple para- 
digms of nursing science. ANS Adv Nurs Sci. 1999; 21(4): 64-80.

5. Fawcett J. Analysis and evaluation of contemporary nursing knowledge: nursing models and theories. 2a ed. Philadelphia: FA Davis Company; 2005.

6. Newman M, Sime A, Corcoran-Perry S. The focus of discipline of nursing. ANS Adv Nurs Sci. 1991; 14(1): 1-6.

7. Triviño Z, Sanhueza O. Paradigmas de Investigación en Enfermería. Cienc Enferm. 2005; 11(1): 17-24.

8. Universidad Nacional de Colombia. Programa de Maestría en enfermería: Modelos conceptuales y teorías de la enfermería: Antologías en CD-ROM Diseños de investigación [monografía en CDROM]. Bogotá: Universidad Nacional de Colombia; 2008.

9. McEwen M, Wills EM. Theoretical basis for nursing. $2 \mathrm{a}$ ed. Philadelphia: Lippincott Williams \& Wilkins; 2007.

10. Durán de Villalobos MM. Enfermería: Desarrollo teórico e investigativo. Bogotá: Unibiblos; 2001.

11. Reed PG, Shearer NC, Nicoll LH. Perspectives on nursing theory. $4 \mathrm{a}$ ed. Philadelphia: Lippincott, Williams \& Wilkins; 2004.

12. Jacox AK, Webster G. Competing theories of science. Comentado en: Nicoll LH. Perspectives on nursing theory. Philadelphia: J.B. Lippincott Company; 1986.

13. Amaro MC. Florence Nightingale la gran teórica de enfermería. Rev Cubana Enferm [Revista en Internet] 2004; 20 (3). Hallado en: http://www.bvs.sld.cu/ revistas/enf/vol20_3_04/enf09304.htm. Acceso el 12 de abril 2011.

14. García ML, Arana B, Cárdenas L, Monroy A. Cuidados de la salud: Paradigma del personal de enfermeros en México - la reconstrucción del camino. Esc Anna Nery Rev Enferm [Revista en Internet] 2009; 13(2). Hallado en: http://www.eean.ufrj. br/revista_enf/20092/artigo\%206.pdf. Acceso el 13 de abril 2011.
15. Ibáñez LE, Sarmiento LV. Proceso de enfermería. Bucaramanga: Universidad Industrial de Santander; 2003.

16. Marriner A, Raile M. Modelos y teorías de enfermería. 6a edición. España: Elsevier; 2007.

17. Grupo de Cuidado Facultad de Enfermería Universidad Nacional de Colombia. Avances conceptuales del grupo de cuidado. En: Grupo de Cuidado y práctica de enfermería de la Universidad Nacional de Colombia. Dimensiones del cuidado. Bogotá: Unibiblos; 1998.

18. Cabrero J, Richart M. El debate investigación cualitativa frente a investigación cuantitativa. Enferm Clin [Revista en Internet] 1996; 6(5). Hallado en: http://departamento.enfe.ua.es/ profesores/miguel/documentos/Debate_ inv-cualitativa_frete-inv-cuantitativa. pdf. Acceso el 12 de abril 2011.

19. Do Prado ML, Schmidt K. Salud y globalización: retos futuros para el cuidado de Enfermería. Invest Educ Enferm. 2004; 22(2). 104-111.

20. Durán de Villalobos MM. Teorías de rango medio: ¿Por qué para el cuidado? Importancia y utilidad para la práctica de enfermería. En: Grupo de Cuidado Facultad de Enfermería Universidad Nacional de Colombia. El arte y la ciencia del cuidado. Bogotá: Unibiblos; 2002.

21. Fawcett J, Garity J. Evaluating research for evidence-based nursing practice. Philadelphia: F.A Davis Company; 2009.

22. Fawcett J. The relationship theory and research. 3a ed. Philadelphia: F.A Davis Company; 1999.

23. Grupo de Cuidado Facultad de Enfermería Universidad Nacional de Colombia. Nuevos avances conceptuales del grupo de cuidado. En: Pinto N, coord. Cuidado y práctica de enfermería. Bogotá: Unibiblos; 2000.

24. Palucci MH. La construcción del conocimiento de la enfermería en el paradigma reflexivo-comprensivo. Rev Lat Am En- 
fermagem. 2006; 14(4): 469.

25. Ailinger RL. Contributions of qualitative research to evidence-based practice in nursing. Rev Lat Am Enfermagem. 2003; 11(3): 275-279.

26. Sánchez B. Fenomenología, un método de indagación para el cuidado de enfermería. En: Pinto N, coord. Cuidado y práctica de enfermería. Bogotá: Unibiblos; 2000.

27. Seminario de Investigación I: Antologías en CD-ROM. Diseños de investigación [monografía en CD- ROM]: Villalobos, MM. Bogotá: Universidad Nacional de Colombia; 2008.

28. Díaz V, Álvaro M, Campo E, Saavedra F, Cabrera MD. El paradigma de la enfermería basada en la evidencia (EBE) en la enfermería clínica hospitalaria. Nure Investigación [Revista en Internet] 2007; 28. Hallado en: http://www.fuden.es/ FICHEROS_ADMINISTRADOR/INV_ NURE/PDF_proyecto2886200710838. pdf. Acceso el 10 de abril 2011.

29. Daza de Caballero R, Torres A, Prieto de Romano G. Análisis crítico del cuidado de enfermería: Interacción, participación y afecto. Index Enferm. 2005; 14(48-49): 18-22.

30. Vásquez M. El cuidado en enfermería desde la perspectiva transcultural: una necesidad en un mundo cambiante. Revista Salus [Revista en Internet] 2000; 4 (3). Hallado en: http://servicio.bc.uc. edu.ve//fcs/index.html. Acceso el 11 de abril 2011. 